\title{
ACUTE HAEMATOGENOUS OSTEOMYELITIS
}

\author{
By Walter Mercer, F.R.S.Ed., F.R.C.S.Ed. \\ Professor of Orthopaedic Surgery, University of Edinburgh
}

\begin{abstract}
No surgical advance in the past ten years has given greater pleasure than to behold the dread spectre of acute osteomyelitis stripped of its terrors. We have seen, in days gone by, the young child carried off by an overwhelming staphylococcal septicaemia, and we have seen unfortunate young adults coming up to our out-patient departments year after year with discharging sinuses and other distressing complications. When it is realized what a change penicillin has brought to this scene it can but make one filled with humble gratitude to the Flemings, the Floreys and the other laboratory workers in the antibiotic field.

Acute osteomyelitis is still a dangerous disease, but both mortality and morbidity have been immensely modified by modern chemotherapeutic drugs. Prior to the successful chemotherapeutic age the mortality was about 25 per cent.; with sulphonamides the mortality fell to ro per cent; now, with penicillin, it is in the region of 2 per cent.
\end{abstract}

\section{The Organism}

By far the most common infecting organism is the staphylococcus pyogenes aureus. Occasionally in the less acute case it is the albus variety. The streptococcus seems to produce multiple lesions. When the infection is carried directly into the bone from septic wounds a greater variety of organisms is likely to be present than in bloodborne infection. The $B$. coli, the $B$. aerogene's capsulatus, and the bacillus of malignant oedema may be found, especially in cases where the original injury was a compound fracture. The pneumococcus is occasionally found and typhoid osteomyelitis is not uncommon. Less frequent organisms are the brucella abortus and the bacillus paratyphosus-A or.B. The B type is eight times more common than the $\mathrm{A}$.

The disease still presents its problems, of course, but I submit that these have been simplified in number to three-how to diagnose osteomyelitis at an early stage, how best to employ penicillin or other antibiotics, and what additional surgical treatment may be employed.

\section{Diagnosis}

Early diagnosis is of great importance because the general experience is that acute osteomyelitis will subside entirely with conservative treatment if penicillin is started within five days of onset of the disease. Between five and ten days most of them go on to abscess formation in spite of penicillin, and after ten days all form abscesses and require some intervention. Early diagnosis is not easy, of course, because for one thing this is now rather a rare disease; so much so that it is estimated that the average general practitioner will see only one case in ten years. The affection appears to have changed its character in recent years in addition to occurring less and less frequently. Jönsson analysed his hospital's cases between I9I2 and I 94 I - a total of 135 cases. If the cases were divided into five-year periods the smallest number of cases was found in the last period, during which there were no deaths. The study revealed, too, that a smaller number of complications occurred during the last five-year period compared with earlier ones. The duration of treatment was considerably shorter during later years and the condition of the patient on admission much better than formerly. In none of these cases was serum or chemotherapy used.

This gradual disappearance of the disease is due to the improved general health of childrendue, no doubt, ts the earlier treatment of debilitating and deficiency diseases, to the slum clearances, better drainage and cleaner streets, and other such important preventative measures.

\section{The Incidence of the Disease}

In spite of this, however, it appears that the condition comes in definite epidemics. Most of the cases are seen in the older child, though it does occur in the newly born, and in them it is usually a generalized process. One is therefore more likely to find in the case of a newly-born child that if there is a staphylococcal infection of bone there will be other staphylococcal lesions elsewhere in the body. The maximum incidence, however, is 
between 3 and 15 years of age. The responsible factors may be the greater liability to trauma and the frequence of a mild bacteraemia at this time of life. Boys are more likely to be affected than girls, in the proportion of about four to one.

\section{The Localizing Influence}

The bones of the leg are more often affected, and of these the tibia suffers most frequently, the upper end being more commonly involved than the lower. In the femur it is the lower end-the area of the trigone-that is the seat of election. The greater area of bone in these regions may explain this incidence while, of course, these epiphyses persist longer and therefore are there to be affected over a greater length of time.

It is generally agreed that a mild bacteraemia precedes the disease, but the reason for its localization in the metaphysis of a long bone is more speculative. The profuse blood supply of this actively growing area must have some influence, and also the ease with which the somewhat loosely attached epiphysis may be damaged, with the production of clots. It is doubtful; however, if trauma plays such an active part as has been considered. It must be very exceptional to find exclusive evidence of trauma, and the history of an accident is probably obtained because acute osteomyelitis is sudden in onset, and it may be that the sudden pain actually causes the child to fall down when the condition is affecting the leg bones.

The blood supply at the margin of the metaphysis is in a series of tortuous loops which are narrow and inelastic and are in reality end arteries. Such an arrangement favours the occurrence of embolism which would in itself cause an anaemia of the area of bone in the immediate vicinity of the metaphysis.

A bacteraemia in such a narrow, tortuous and inelastic arrangement may cause a localized swelling of the lining of the vessels, a thrombosis may occur, or there may be an embolus, but all these will block the stream. In such circumstances a devascularization of an area of bone in the metaphysis occurs, and in addition there is a stagnant stream of infected blood, and so the stage is set, and ideally so, for the formation of an abscess as the first stage in the acute haematogenous osteomyelitis.

Besreda pointed out years ago that the tissues of the body have receptor cells in which organisms become localized irrespective of their route of entry. It may be that there is a receptor area for pyogenic organisms in this area, probably situated in the reticulo-endothelial system. The reticuloendothelial depots of the marrow are situated at the metaphyseal ends of the long bones and it is precisely here that the human focus of infection occurs.

\section{Pathology}

Once the infected blood becomes closed up, $\subseteq$ either by thrombosis or by the oedema of trauma $\vec{B}$ closing the exits and entrances of the area, the bacteria multiply rapidly and a small-at first localized abscess results. This enlarges from the

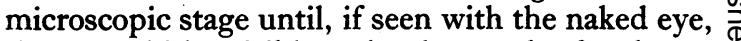
there would be visible a circular patch of oedematous, congested and purulent marrow. Although कै it is sometimes said that there is a localized osseous $\overrightarrow{0}$ type and a generalized septicaemic type, it is unlikely that this is true; both are merely degrees of $\vec{\omega}$ the same disease and enormous variations in the severity of it do occur. Some cases belong to the fringe of the disease, run a mild course and may not even come into the category of acute osteomyelitis. Depending to some extent on the type and virulence of the infection and no doubt, too, on the fertility or otherwise of the soil, and without treatment, the disease may take one of $\mathrm{O}$ several courses:-

(a) If the resistance is strong and the infection a mild one, the reaction on the metaphyseal area $\stackrel{\complement}{<}$ may be powerful enough to localize, kill and $\vec{\theta}$ finally eradicate the organisms before suppuratio 8 o occurs.

(b) In slightly less favourable conditions, eithe⿳亠丷厂 from a less powerful resistance or a more virulent organism, the reaction may still be enough to localize but not to eradicate the infection, and a chronic localized area of suppuration, or a Brodie's abscess, may form.

(c) In the third degree of variation the usual sequence of changes may occur, the initial small area of congestion proceeding to suppuration and sequestration.

(d) The bacteraemia may become a septicaemia with the formation of multiple abscesses in the lungs, kidney, liver and bones.

(e) In the case of extreme virulence and diminished resistance or none, there will be little or no localization and the whole picture is one of a generalized acute septicaemia.

When the disease follows either of the first two courses the acute illness gradually subsides. Should a Brodie's abscess be formed the organisms sometimes retain their vitality for a considerable time and under certain circumstances may undergo a recrudescence of activity. In the centre of the cancellous tissue a cavity develops filled with thick pus, and owing to the chronic nature of the disease the surrounding bone is sclerosed and condensed. The chronic type of disease, it is hoped, will soon be a rarity, and sequestration, cloacae and chronic discharging sinuses may be but names. 
It is not necessary to detail all the signs and symptoms, but because of the importance of early recognition and accurate presumptive diagnosis I wish to emphasize the significant symptom, which is pain, and the conspicuous sign, which is tenderness.

The pain is complained of at a very early stage and is over the infected area, which is usually the metaphysis of a growing long bone and, in 70 per cent of cases, of the tibia or femur. The sign is a constant and exquisite tenderness elicited with the tip of the finger over the most easily accessible point on the bone-the so-called finger point tenderness. Swelling of the soft parts and warmth appear later and there is little else to be made out locally. In the acute fulminating or septicaemic type, now a comparatively rare occurrence, the local signs are overshadowed by evidences of extreme toxaemia with high fever, delirium, sleeplessness, etc, and the above sign can be found only by the most meticulous examination. However, if acute osteomyelitis is kept in mind, it is unlikely that the diagnosis will be missed.

Acute rheumatic fever, acute poliomyelitis and acute arthritis may be diagnosed in error. Careful examination in acute rheumatic fever will localize the tenderness over the joint and it is diffuse. In an acute case with many joints affected, the cause need not necessarily be the arthritis of rheumatism, for in acute cases of haematogenous osteomyelitis. there may not infrequently be several foci, and the fallacy of salicylic acid being given from the diagnostic aspect in such cases is obvious.

In poliomyelitis there are many points of contrast, but in particular the tenderness is in the muscle rather than the bone. Septic arthritis may be difficult to diagnose and careful examination of the joint and possibly aspiration are usually helpful.

It is, of course, necessary when taking $\mathrm{X}$-rays of the affected part that there should always be a comparable picture taken of the other limb in order to show minute differences. One cannot too strongly emphasize that a radiogram at this early stage is of relatively little value, the only signs at this stage being the appearance of oedema of the soft tissues at the site of the disease. This is manifested by a swelling of the surrounding muscles and by blurring or obliteration of the fatty intermuscular septa. In a somewhat later stage oedema of the subcutis, which results in this layer appearing more indistinct and becoming broader than on the healthy side, is sometimes noticed as well. If the change affects a joint a distension of the soft tissues of the joint with an increase in the joint fluid is noticed as well. In acute septic infection of the hip joint an infiltration is ob- served in the soft tissue on the inner side of the pelvis. One can then observe on the diseased side a more or less increased breadth of the soft tissue shadows proceeding down and along the inner edge of the pelvis. X-rays showing a higher degree of contrast of the different soft tissue structures are necessary. Soft tissue films are therefore of some value. In an untreated case bone changes are rarely present before the tenth day, while in cases treated with penicillin they may be delayed till the third or fourth week.

I have been interested lately in trying to determine whether a case of acute osteomyelitis that has settled down on conservative treatment always shows subsequent $\mathrm{X}$-ray changes in the bone. And if the bone subsequently appears perfectly normal, how can we be sure that the case really was one of osteomyelitis? It is perhaps an academic point, but it is important scientifically when we are considering statistics of the disease. In my experience, the great majority do show subsequently X-ray evidence of past disease, and from a study of my own cases and of those of colleagues in Edinburgh, I had become sceptical of the original diagnosis in the absence of X-ray change. Nevertheless, I am inclined to concede that it can happen occasionally. I recently saw $\mathrm{X}$-rays of a typical such case where a competent surgeon had no doubt about the clinical diagnosis and, moreover, where the blood culture had been positive.

In this connection Altmeir published an interesting review of radiograms of the disease. He divided his cases into four groups:-

Group I. The correct diagnosis was made within the first two days and adequate treatment started immediately. In these the results were excellent and the bone changes minimal, consisting of areas of localized periosteal reaction, small areas of decalcification and little or no evidence of sequestration. In a few the $\mathrm{X}$-ray findings were entirely negative.

Group 2. The diagnosis and treatment were moderately delayed and some had soft tissue abscesses. In these, after a week or so, periosteal reaction and patchy demineralization became evident in the $\mathrm{X}$-ray and these were most marked in one to five months.

Group 3. Delay in the diagnosis and treatment occurred in this group up to seven to ten days. There was severe bone destruction, soft tissue abscess and sequestration. X-rays in these showed extensive bone destruction at the start of penicillin therapy. This increased at further examinations. Small sequestra were absorbed and larger ones acted as autogenous grafts. The sequestra were rarely discharged.

Group 4. This was of a fulminating character 
and surgical intervention still constituted a surgical emergency! In this group the bone destruction was very extensive, due to the severity of the infection.

Accurate diagnosis, apart from the presumptive, is made when the child is admitted to hospital. But since penicillin has often been started already, and rightly so, in suspected cases, the blood culture is often negative. However, in all suspected cases, treated or not, immediate culture of the blood for organisms is made and their sensitivity to penicillin ascertained, and a leucocyte count and the determination of the erythrocyte sedimentation rate are carried out. If the result of the blood culture is positive it is wise to repeat it daily until the blood is sterile. If the result of the first culture is negative and the temperature remains elevated a further culture is made. The leucocyte count and the determination of the sedimentation rate are repeated at two- to three-day intervals. Where there is an abscess this is aspirated to identify the organism and to test its sensitivity. A routine $\mathrm{X}$-ray is done to exclude other possibilities.

\section{Treatment}

Penicillin. The pathological basis for treatment of acute haematogenous osteomyelitis is two-foldfirst, the generalized infection must be overcome to save life; and second, the bone lesion must be controlled. When the causative organism is sensitive to the antibiotics, amelioration of the general disease can be expected. 'The bone lesion is an inflammation within a rigid compartment. When this process is advanced, circulation is impaired and bone necrosis results. Specific antibacterial treatment, given in adequate doses while the circulation is still unimpaired, should lead to early recovery. Once pressure and thrombosis have blocked the circulation the site of infection becomes less accessible to the therapeutic agent and the results must be less favourable.

This is a septicaemic state and therefore it is essential to give penicillin in full systemic dosage; that is, the penicillin blood level must never be allowed to fall below o.r unit per cc.-the level required to inhibit the staphylococcus aureus.

A level of 0.1 unit per cc. is achieved by 15,000 units three-hourly, and this will be sufficient to inhibit any staphylococci circulating in the blood stream. But in osteomyelitis the organisms are shut away in dense tissue and rigid compartments, and so the dosage must be considerably higher so that at least some of the antibiotic will arrive at the infected area. It is therefore suggested that the initial dose be 200,000 units followed by 50,000 units three-hourly.

I have also been quite satisfied with a regime of once-daily injections of 300,000 units procaine penicillin and 100,000 units crystalline sodium penicillin, combined with aluminium stearate in arachis oil. This provides a high initial concentration of penicillin in the blood and a subsequent inhibitory level for 24 hours. In the few cases in which $I$ have tried it this regime has given results similar to that which I would have expected with three-hourly injections.' At any rate, for the first week of treatment it is necessary to have a generous systemic cover, and it is also necessary to continue, with rather smaller dosages, for a second and sometimes third week of treatment.

Penicillin dosage such as this should be quite adequate for the staphylococcal infection of acute osteomyelitis, and I am opposed on principle to fantastically high doses, such as are sometimes employed. I deprecate such doses because I am impressed with the laboratory work of Garrod, Eagle and others, who have shown that as far as the staphylococcus aureus is concerned there is an optimum level of penicillin concentration, and it is in the region of 0.1 unit per cc. Eagle has called this the 'zone phenomenon,' and shown that there is no advantage in increasing this concentration a thousand-fold and, indeed, the bactericidal effect may be reduced by doing so. I cannot say whether, in fact, any cases of acute osteomyelitis have done less well on enormously high dosages, but I think that we should certainly pay attention to such careful studies by our laboratory colleagues.

We must also be constantly on the watch for the penicillin-resistant organism. Mary Barber has reported an incidence of 59 per cent. of resistant strains in the wards of Hammersmith Hospital, and I have found similar figures in my own wards in Edinburgh. This is probably due to crossinfection in the wards and the persistence of the resistant strains, but fortunately in out-patients, not the victims of ward cross-infection, the figure is not higher than 5 or Io per cent. Nevertheless, 5 to ro per cent. of cases of acute osteomyelitis may be caused by a staphylococcus resistant to penicillin, and therefore it is important that the organism obtained fiom the blood culture, or fiom the abscess, should be tested for sensitivity. If there is no opportunity of testing for sensitivity, then careful clinical re-assessment must be made frequently, and if temperature and pain are not relieved in an early case after 48 to 72 hours, a switch should be made to another antibiotic. In the present state of our knowledge, aureomycin is probably the second drug of choice, 10 to $60 \mathrm{mg}$. per kilo body weight orally per day. The Mayo Clinic has reported successful treatment by aureomycin of septicaemias due to penicillin-resistant staphylococci. 


\section{Operative Treatment}

In all cases the limb must be promptly immobilized, preferably in plaster with a wide window for observation, and general supportive measures instituted-sedatives for pain and the maintenance or, where necessary, restoration of the blood fluid and electrolytic levels. Actual operative intervention is unlikely to be needed in the early cases where penicillin has been started in the first five days of the disease. In the relatively. late cases-after ten days-operation is almost always necessary to deal with the abscess and necrotic bone. It is in the intervening groupwhere there is probably a subperiosteal abscessthat there is some doubt about the proper method of intervention. Various methods have their adherents-aspiration and irrigation with penicillin; intramedullary injection; incision of abscess and closure, etc.; and from all of the methods there have been reports of successfully treated cases. I do not think ought to be dogmatic about what is the best method of operative treatment-the important thing is that the treatment should be meticulously carried out by an experienced surgeon who has confidence in his own method and who appreciates the whole problem. My preference is for that method in which the subperiosteal abscess is incised, the bone drilled and the wound closed. Trueta has pointed out that penicillin cannot sterilize an abscess and that, if it is left, the disease in the bone may progress and lead to extensive destruction. An intramedullary abscess produces thrombosis of the arterioles and a severe and local oedema. These induce a spread of the abscess and eventually an extensive necrosis of bone which is walled off from the circulating blood. It is an old surgical principle that where there is pus it should be let out, and when this is carried out in the treatment of osteomyelitis with penicillin the latter gets a better chance to act. The removal of pus allows the oedema to subside and so allows systemic penicillin to reach the marrow cavity and this prevents further formation of pus. To prevent secondary infection the wound is immediately sutured. In all such cases, therefore, the abscess is opened and a tourniquet used where the position permits it. An abscess in the soft tissues implies an intramedullary abscess and therefore the bare bone is drilled with a 6 to $8 \mathrm{~mm}$. drill in several places, and invariably oily greenish pus exudes through the holes. A local injection is made with a syringe into the drill holes of 100,000 units of penicillin in 5 to ro cc. distilled water, a few tablets of pure penicillin are left outside the bone and the wound completely stitched up in two layers if possible. It is most important to do a primary suture, for the so-called open treatment almost invariably is followed by a secondary infection, delayed healing and a tendency to recurrence. The limb is then immobilized in a plaster-of-paris case and a moderately-sized window cut to observe the area. It is unlikely that further local treatment will be required and the immobilization of a complete cast, rather than the use of a bivalved one, is more efficient.

If the infection is in the neck of the femur or in the acetabulum, and particularly if the initiation of treatment is delayed, it is most important to put traction on the leg for several weeks. The affected area of bone is softened by the disease so that the powerful thigh muscles will easily separate the head of the femur or crush the soft bone and infect the joint. Such traction is best carried out by using the plaster spica and fixing it to the foot of the bed, which is then elevated on blocks.

In conclusion, I believe, as do so many, that by the development of antibiotics the tragedy of osteomyelitis has been overcome. The fault is ours if the tragedies of an earlier surgical day are not abolished for all time. It may be that we are apt to become complacent and sit back on our treatment. We must guard against this tendency and be ready to make full clinical re-assessment at any. time. I believe, however, at the present time that early diagnosis, energetic chemotherapy and judicious surgery should lead to rapid cure without deformity and devoid of unpleasant sequelae. 